\title{
Design of Articulated Leg-Wheel Subsystem by Kinetostatic Optimization
}

\author{
Aliakbar Alamdari ${ }^{1, *}$ \\ Mechanical and Aerospace Engineering \\ The State University of New York at Buffalo \\ Email: aalamdar@buffalo.edu \\ Venkat N. Krovi \\ Mechanical and Aerospace Engineering \\ The State University of New York at Buffalo \\ Email:vkrovi@buffalo.edu
}

\begin{abstract}
High mobility, maneuverability and obstacle-surmounting capabilities are highly desirable features for rough-terrain locomotion systems. In this paper, we explore the use of various candidate articulated leg-wheel subsystem designs (based on the four-bar mechanism) to enhance locomotion capabilities of land-based vehicles. Multiple leg-wheel design parameters, such as kinematic link lengths and static spring stiffnesses and preloads, influence the overall locomotion performance. Appropriate selection can not only enhance the robot climbing performance but also reduce the wheel slip as well as the overall energy consumption.

In particular, we aim to: (i) achieve the greatest motion-ranges between wheel-axle and chassis; as well as to (ii) reduce the overall actuation requirements by spring assist. Hence, we explore the use of systematic kinetostatic design approaches coupled with optimization to determine the parameters for alternate leg-wheel subsystem designs. Further, we also examine enhancement of uneven-terrain locomotion by varying subsystem parameters during the terrain traversal via a semi-active leg-wheel subsystem. Extensive simulation is then employed to evaluate the capabilities of these alternate articulated leg-wheel designs to surmount a predetermined/sensed terrain traversal profile while reducing actuation requirements.
\end{abstract}

Keywords: Kinetostatic synthesis, Design parameter optimization, Articulated leg-wheel subsystem.

\section{Introduction}

Over the past decade, there has been a growing interest among researchers in land-based locomotion systems for operation on unstructured terrain with increasingly diverse applications such as rough-terrain planetary explorations $[1,2]$, rescue operation [3] and disaster environments [4]. The mechanical design architectures explored for mobility on rough terrain vary considerably. In general, wheeled systems perform fast, smooth and power- efficient on flat surfaces [5]. In contrast, articulated legged systems such as quadruped Titan series [6] provide greatest agility/mobility on rough and unstructured environments. Hybrid articulated leg-wheel vehicles [7] (shown in Fig. 1) offer an interesting via-media to exploit the benefits of both legged and wheeled systems.

Previous work on hybrid articulated vehicles had examined systems with both active and passive suspensions. Rovers with leg-wheel subsystems range from: (i) vehicles with rocker bogie suspensions like Sojourner [8], Rocky 7 [9], Micro5 [12], Shrimp [10], NOMAD [13]; (ii) designs with articulated frames which can typically overcome obstacles of their wheel size such as Wheeled Actively Articulated Vehicle (WAAV) [14, 15, 16]; to (iii) systems with powered legs and active/passive wheels such as Work Partner [17], Roller-Walker [18], and ALDURO [19]. In the

\footnotetext{
${ }^{*}$ Corresponding author

Email address: aalamdar@buffalo.edu (Aliakbar Alamdari)
} 
passive cases, as illustrated in Fig. 1(a), the suspension system accommodate passively to the uneven terrain without any actuation for controlling the internal configuration of the vehicle. In turn, the traction forces at the wheel-ground contact also adjust passively during the terrain traversal. Rocky7 [9] and Shrimp [10] are examples of such passive vehicles. On the other hand, articulated vehicles with active suspension systems, Fig. 1(b), require actuation to change configuration and often even to maintain static stability under gravity. Various articulated leg-wheel designs (with multiple revolute/prismatic joints between the wheel and the chassis) could be realized depending on the type, number and sequence of the joints. Adding additional articulation increase the intermediate degree of freedom within the chain, which need to be controlled or restricted either: (i) actively by actuation, (ii) semi-actively using springs and dampers [20]; or (iii) passively by adding some form of structural equilibration using hardware constraints.

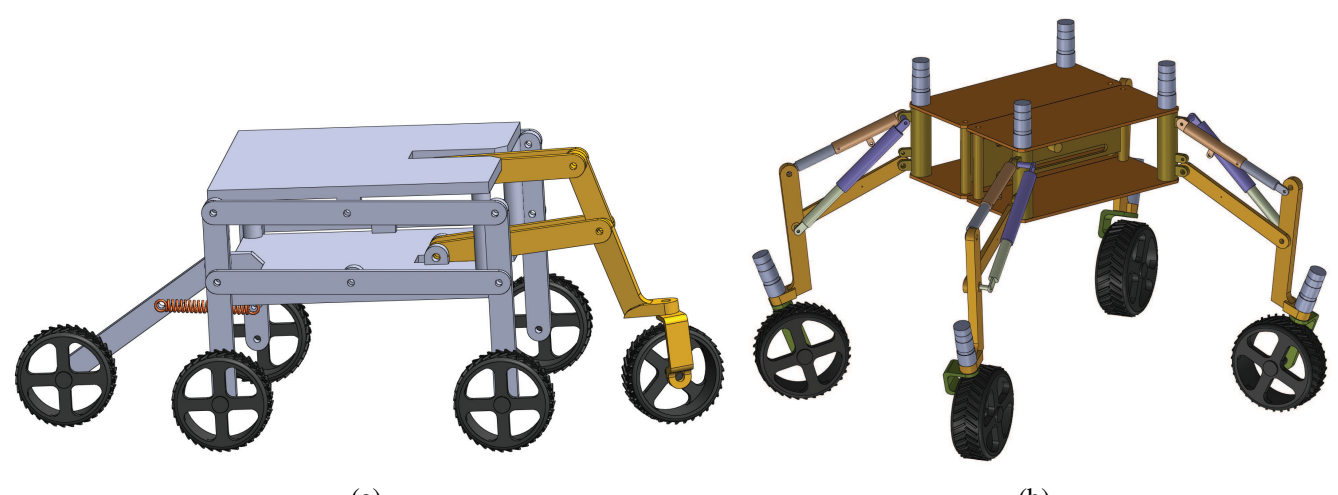

(a)

(b)

Figure 1: (a) Passive articulated leg-wheel vehicle, (b) Highly reconfigurable articulated wheeled vehicle with four actuators in each leg-wheel subsystem for maneuvering on uneven terrains [20]

In addition to foremost necessities such as high mobility, obstacle-surmounting capability and maneuverability, there are additional criteria such as robustness, reliability, and efficiency need to be considered in the design process. In almost all cases no systematic effort to design the articulated leg-wheel system is ever considered. However, to the authors best knowledge, very few publications can be found in the literature that discuss the application of formal mechanism design principles of the leg-wheel subsystems. For instance, a stair-climbing mobile platform built on the Rocker-Bogie mechanism is able to climb up/down various sizes of stairs [21]. In this paper, link parameters were optimally chosen via Taguchi method in order to make the trajectory of the center of mass as smooth as possible. However, they do not consider use of spring-assists to control the internal configuration of the front leg or normal forces in the front wheel. Singh et. al. [22] proposed use of a novel compliant rover for rough terrain mobility. Their approach optimizes the torsion spring parameters at one of the joints in the rover's suspension to control the internal configuration of the vehicle. However, no efforts at systematic kinematic and static synthesis of the leg-wheel subsystem were explored to determine link length/torsion spring parameters.

The key contribution of this paper is the systematic exploration of the kinetostatic mechanism design process to articulated leg-wheel subsystems. We propose and examine multiple candidate leg-wheel subsystem designs and the development of an optimization framework to enhance terrain accommodation and reduce the traction force requirements. The load-bearing requirements can be significant and are highly configuration dependent- we seek to exploit structural equilibration by internal reconfiguration to bear these loads. To this end, we enhance the kinetostatic optimization to allow for structural reconfiguration planning. The kinematic and static synthesis of the leg-wheel subsystem is carried out to determine the optimal link length/torsion spring parameters. We also explore the benefits of employing semi-active leg-wheel designs, with varying link length/spring constants along the path to maximize the traction forces at the wheel-ground contact point. Comparative studies were performed between the rigid suspension vehicle and alternate compliant suspension systems to illustrate the effect of compliance in improving stair climbing via extensive simulations.

The rest of this paper is organized as follows: In section 2, we provide a brief background of the general kinematic and kinetostatic design approaches used for mechanism design. In section 3, we specialize the kinematic/kinetostatic approach for leg-wheel subsystem with variable-length crank link to: (i) surmount step profiles, and (ii) reduce ac- 
tuation requirements. In section 4, alternate designs are presented and compared, and section 5 presents concluding remarks.

\section{Background}

\subsection{Kinematic Synthesis}

Dimensional/ Kinematic synthesis is the process of determining the geometric parameters of the mechanism that will perform a specific task. In here, geometry determination comprises selection of the lengths of the individual links that make up the mechanism to perform a desired function generation, path generation, and motion generation [24]. For these three synthesis types, the prescribed conditions that the mechanism must satisfy are called the "precision points". The resulting linkage can create the desired motion precisely at precision points and approximately at other positions. Increasing the number of precision points allows closer tracking of the ideal desired path, but is more nonlinear and difficult to solve. Fortunately, many real world problems only need few critical positions to be satisfied precisely. A fixed-length four-bar linkage can match five motion-generation precision points and nine path following precision points exactly. The adjustable four-bar linkage, such as the one we will explore, on the other hand, can satisfy many more precision points by making some of the parameters adjustable.

Optimal synthesis methods minimize an error function of the difference between the generated and the desired motions, called the Structural Error, to find the mechanism that gives the best approximation of the desired motion. For path following, structural error has been conventionally defined as the sum of squares of the distances between appropriately selected correspondence points desired and the generated paths.

\subsection{Kinetostatic Synthesis}

One of the main issues which have the most effect on the performance of leg-wheel design is the force interaction between a linkage and its environment. The kinetostatic synthesis is used to assist the selection of the optimal configuration (and determine the minimum torques required) to support these external loads by structural equilibration. The goal of the articulation is to guide the attached wheel through several positions while supporting a set of specified external loads. We also add torsion springs at each joint to minimize/optimize the peak actuator torques by a suitable combination of structural equilibration design and spring assists. The formulation and simultaneous solution of the kinematic constraint and static equilibrium equations at the precision points aids the process of simultaneous determination of optimal mechanism and spring parameters [23].

\section{Design Process of Leg-Wheel Subsystem}

In this section the systematic design process of articulated leg-wheel subsystem is presented. Two broad variants of four-bar based articulated leg-wheel systems are examined in Fig. 2: (i) Fixed-length and (ii) Variable-length systems [26]. Further, each of these broad categories are studied with (i) torsional/linear spring assists; and (ii) no spring assist in the leg-wheel subsystem. Finally, in the leg-wheel mechanism group with spring assist, each link i.e., crank, coupler, ground or rocker could be replaced with a linear spring-damper.

From the analysis perspective, the relationship between joint variables $\left(\theta_{2}, \theta_{3}\right.$ and $\left.\theta_{4}\right)$ and Cartesian variables $(x$ and $y$ ) of the leg-wheel subsystem is determined from the differential articulated kinematics. Then, the quasi-static modeling and required constraints for no-slip motion are applied. Next, an objective function based on the discrepancy between the desired and actual wheel-axle path is defined to aid the dimensional synthesis. Finally, the objective function to reduce actuation requirements by optimizing over the kinematic and static parameters is developed.

\subsection{Differential kinematics of the variable-length crank link}

The loop-closure equation of the leg-wheel subsystem with a four-bar based mechanism and variable-length crank link [26] (semi-active crank link), depicted in Fig. 3(a), is written as follows:

$$
\begin{aligned}
\rho z_{2} \cos \theta_{2}+z_{3} \cos \theta_{3} & =z_{4} \cos \theta_{4} \\
\rho z_{2} \sin \theta_{2}+z_{3} \sin \theta_{3} & =-z_{1}+z_{4} \sin \theta_{4}
\end{aligned}
$$




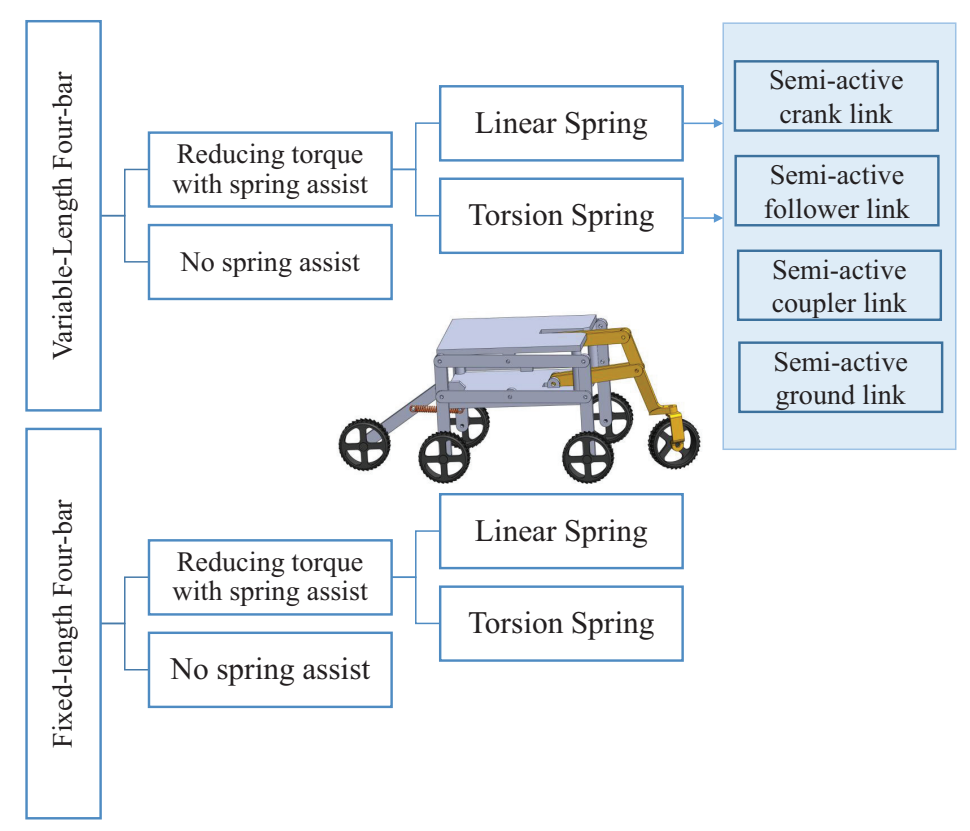

Figure 2: Candidate designs for articulated leg-wheel subsystem.

where $z_{1}, z_{2}, z_{3}$ and $z_{4}$ are the length of ground, crank, coupler and rocker link, respectively. $\theta_{2}=\Theta_{2}+\alpha, \theta_{3}=\Theta_{3}+\beta$ and $\theta_{4}=\Theta_{4}+\gamma$; such that parameters $\Theta_{i}$ are initial absolute angle of $i$ th link and $\alpha, \beta$ and $\gamma$ are incremental angular displacements of link 2, 3 and 4 relative to the initial configuration, respectively; and $\rho$ is the stretch ratio of crank link.

The dependent virtual angular displacements of $\theta_{3}(\delta \beta)$ and $\theta_{4}(\delta \gamma)$ can be expressed in terms of the independent virtual angular displacement of $\theta_{2}(\delta \alpha)$ and the virtual linear displacement of $\delta \rho$ as follows:

$$
\left[\begin{array}{l}
\delta \beta \\
\delta \gamma
\end{array}\right]=\left[\begin{array}{cc}
z_{3} \sin \left(\theta_{3}\right) & -z_{4} \sin \left(\theta_{4}\right) \\
-z_{3} \cos \left(\theta_{3}\right) & z_{4} \cos \left(\theta_{4}\right)
\end{array}\right]^{-1}\left[\begin{array}{cc}
-\rho z_{2} \sin \left(\theta_{2}\right) & z_{2} \cos \left(\theta_{2}\right) \\
\rho z_{2} \cos \left(\theta_{2}\right) & z_{2} \sin \left(\theta_{2}\right)
\end{array}\right]\left[\begin{array}{l}
\delta \alpha \\
\delta \rho
\end{array}\right]=\left[\begin{array}{ll}
C_{11} & C_{12} \\
C_{21} & C_{22}
\end{array}\right]\left[\begin{array}{l}
\delta \alpha \\
\delta \rho
\end{array}\right]
$$

The virtual displacement of wheel-axle caused by forces $F_{x}, F_{y}$ and moment $M_{z}$, i.e., $\delta x$ and $\delta y$ and $\delta \phi$, respectively (as shown in Fig. 3(b)), can be derived by differentiating the wheel-axle pose in X-Y coordinate attached to the chassis as follows:

$$
\begin{aligned}
& \delta x=\left[\begin{array}{ll}
-\rho z_{2} \sin \left(\theta_{2}\right)-z_{6} \sin \left(\theta_{3}\right) C_{11} & z_{2} \cos \left(\theta_{2}\right)-z_{6} \sin \left(\theta_{3}\right) C_{12}
\end{array}\right]\left[\begin{array}{l}
\delta \alpha \\
\delta \rho
\end{array}\right]=\left[\begin{array}{ll}
C_{31} & C_{32}
\end{array}\right]\left[\begin{array}{l}
\delta \alpha \\
\delta \rho
\end{array}\right] \\
& \delta y=\left[\begin{array}{ll}
\rho z_{2} \cos \left(\theta_{2}\right)+z_{6} \sin \left(\theta_{3}\right) C_{11} & z_{2} \sin \left(\theta_{2}\right)+z_{6} \cos \left(\theta_{3}\right) C_{12}
\end{array}\right]\left[\begin{array}{l}
\delta \alpha \\
\delta \rho
\end{array}\right]=\left[\begin{array}{ll}
C_{41} & C_{42}
\end{array}\right]\left[\begin{array}{l}
\delta \alpha \\
\delta \rho
\end{array}\right] \\
& \delta \phi=-\delta \beta=-\left[\begin{array}{ll}
C_{11} & C_{12}
\end{array}\right]\left[\begin{array}{l}
\delta \alpha \\
\delta \rho
\end{array}\right]
\end{aligned}
$$

Note that $z_{6}=z_{3}+z_{5}$ in these equations. The virtual displacement of the mass center of each link can be expressed 


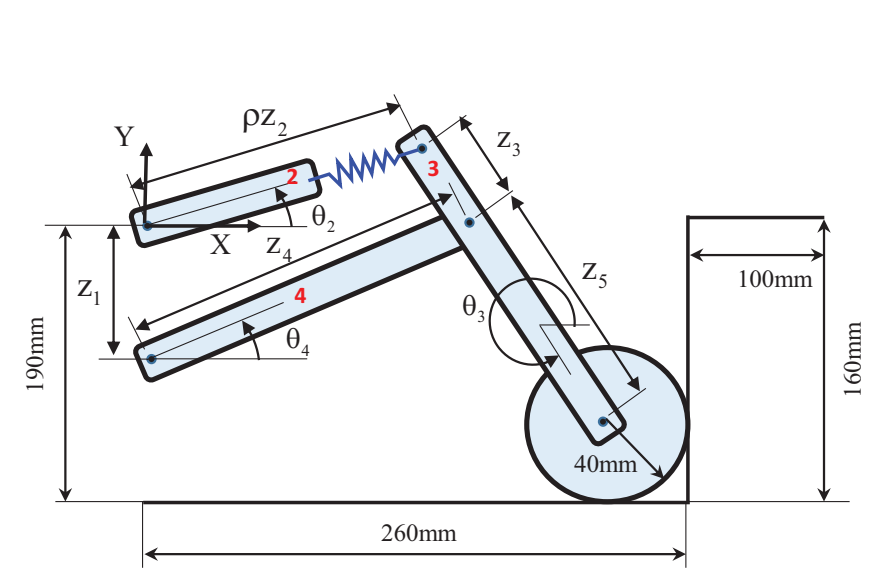

(a)

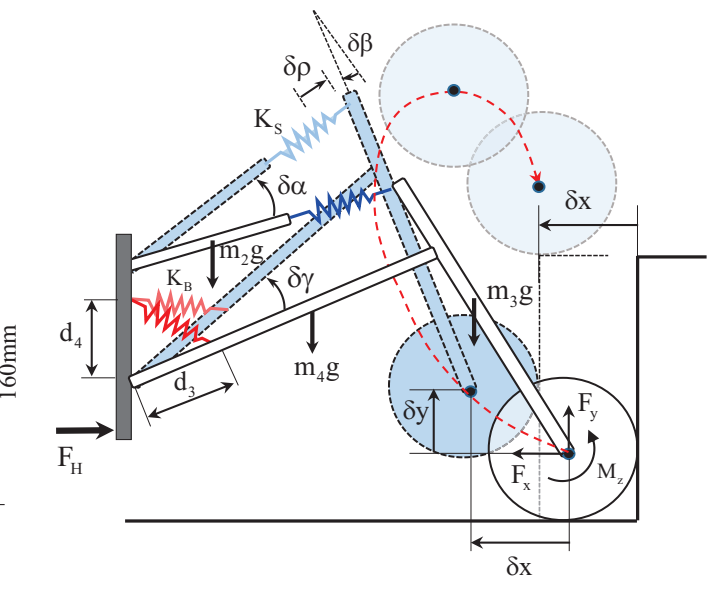

(b)

Figure 3: (a) Geometric parameters of leg-wheel subsystem, step profile and initial position of wheel-axle in X-Y coordinate attached to chassis (in $\mathrm{mm}$ ), (b) Applied forces and virtual displacement of the leg-wheel subsystem

in terms of the independent virtual displacement $\delta \alpha$ and $\delta \rho$ as:

$$
\begin{aligned}
& \delta y_{2}=\left[\begin{array}{ll}
\rho z_{2} \cos \left(\theta_{2}\right) / 2 & z_{2} \sin \left(\theta_{2}\right) / 2
\end{array}\right]\left[\begin{array}{l}
\delta \alpha \\
\delta \rho
\end{array}\right]=\left[\begin{array}{ll}
C_{51} & C_{52}
\end{array}\right]\left[\begin{array}{l}
\delta \alpha \\
\delta \rho
\end{array}\right] \\
& \delta y_{3}=\left[\begin{array}{ll}
\rho z_{2} \cos \left(\theta_{2}\right)+z_{6} \cos \left(\theta_{3}\right) C_{11} / 2 & z_{2} \sin \left(\theta_{2}\right)+z_{6} \cos \left(\theta_{3}\right) C_{12} / 2
\end{array}\right]\left[\begin{array}{l}
\delta \alpha \\
\delta \rho
\end{array}\right]=\left[\begin{array}{ll}
C_{61} & C_{62}
\end{array}\right]\left[\begin{array}{l}
\delta \alpha \\
\delta \rho
\end{array}\right] \\
& \delta y_{4}=\left[\begin{array}{ll}
z_{4} \cos \left(\theta_{4}\right) C_{21} / 2 & z_{4} \cos \left(\theta_{4}\right) C_{22} / 2
\end{array}\right]\left[\begin{array}{l}
\delta \alpha \\
\delta \rho
\end{array}\right]=\left[\begin{array}{ll}
C_{71} & C_{72}
\end{array}\right]\left[\begin{array}{l}
\delta \alpha \\
\delta \rho
\end{array}\right]
\end{aligned}
$$

\subsection{Quasi-static modeling of leg-wheel subsystem with variable-length crank link}

The quasi-static modeling of the leg-wheel subsystem with semi-active crank which is replaced instead of rigid crank link in four-bar based mechanism (as shown in Fig. 3(b)) is presented here. Proper geometric parameters and choosing proper spring stiffness not only reduces the wheel slippage but also reduces the overall energy consumption, and increases the robot's climbing performance. Thus, good distribution of torques is critical to fulfill this goal. It is noteworthy to find the governing equation on wheel slip and explain which function should be minimized to reach this goal.

Figures 3 and 4(a) depict the mechanical structure and applied forces/moments on the leg-wheel subsystem. We note that forces and moments at the end-effector are intimately dependent upon the motor torques applied on the wheel-axle. We assume that the chassis weight creates a vertical force $W_{\text {leg }}$ at the axle, as shown in Fig. 4(a), and the motor mounted at the wheel-axle exerts a torque of $\tau_{\text {motor }}$ on the wheel. Here, it is also assumed all six wheels with radius $R$ are similar. The horizontal force $F_{H}$ generated from the rear wheels can be written as summation of all traction forces i.e. $F_{H}=5 T_{r}=\frac{5 \tau_{r}}{R}$, which here is assumed as known value. Thus, from Figs. 3 and 4 (a) and having quasi-static condition, the resultant forces/moments at the wheel-axle i.e., $F_{x}$ and $F_{y}$ and moment $M_{z}$ easily is obtained as:

$$
F_{x}=N-\frac{5 \tau_{r}}{R}, F_{y}=T-W_{w}-W_{l e g}, M_{z}=-\tau_{m o t o r}
$$

The normal force $N$ is critical factor for the vehicle to surmount the obstacle, because the traction force $T$ is a function of normal force $N$. Therefore, the wheel will always be in contact with surface of the obstacle. When the front wheel is climbing the step like obstacle, the normal reaction force from the obstacle is horizontal and the weight of vehicle and leg-wheel does not has any component in horizontal direction. With increasing the value of normal force, the no-slip margin increases and then larger values of torques must be commanded to the motor. Eq. 
(9) means that the normal force in the front leg-wheel arises from the traction forces at the rear wheels $T_{r}$ and force from reconfiguration of four-bar mechanism due to spring effects $F_{x}$ (both linear and torsion springs). When the front wheel rotates and moves forward, deform springs, the reconfiguration and deformation in the springs generates the additional reaction force at the surface which is named $F_{x}$ here.

When the wheel is climbing the obstacle the rear wheels have maximum probability of slipping. This slippage yields the loss of transmission of traction forces from the rear to the front wheels. Therefore, the slip should be minimized on all the wheels. Since in this paper the focus is on front leg-wheel design, we assume the other five rear wheels are on the flat surface and each causing the traction force $T_{r}$.

Here, the friction coefficient is used to evaluate the climbing capability of the mobile robot. Therefore, in order to avoid wheel slip, the friction force, which directly depends on the motor torque $\tau_{\text {motor }}$, must satisfy Coulomb friction law as $T=\frac{\tau_{\text {motor }}}{R} \leq \mu_{0} N$ where $\mu_{0}$ is the real static friction coefficient.

In order to avoid wheel slip, the virtual friction coefficient $\mu^{*}=\frac{T}{N}$ is defined to examine the risk of slippage. With minimizing this ratio we reduce the possibility of slippage. Therefore, it is highly desirable to minimize the $\mu^{*}$ to reduce the risk of slip without knowing the real static friction coefficient $\mu_{0}$. This goal can be achieved by maximizing the traction forces of six controllable torque inputs of the vehicle which is equivalent to minimizing the function $\max \left(\frac{T}{N}\right)$ for the wheels [25]. The traction and normal forces are in terms of design parameters i.e., configuration and spring parameters. The result of this optimization problem leads to optimum values for geometric and static parameters.

$$
\min \left(\max \left(\frac{T}{N}\right)\right)
$$

Subject to a set of inequality constraints given by:

(i) $N>0$, which corresponds to the constraints which the wheel always maintains in contact with ground.

(ii) $T \leq \mu_{0} N$ which corresponds to no-slip constraints.

(iii) $\tau_{\text {motor }}^{\text {min }} \leq T R \leq \tau_{\text {motor }}^{\text {max }}$, which corresponds to a torque required to generate the traction must be between $\tau_{m o t o r}^{\text {min }}$ and $\tau_{\text {motor }}^{\max }$.

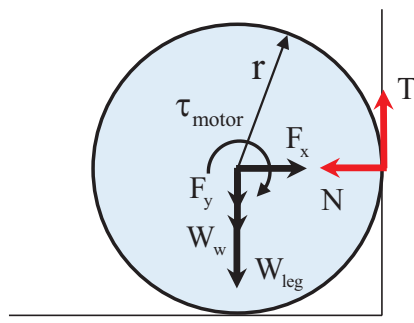

(a)

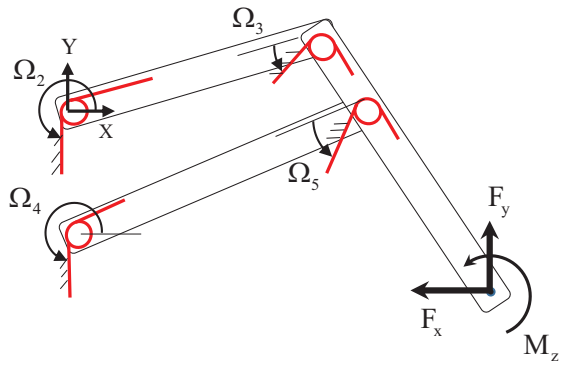

(b)

Figure 4: (a) Applied forces/moments on the wheel, (b) Leg-wheel subsystem with torsion spring at the joints to improve the climbing capability

The above optimization problem is solved for the values of traction and normal forces with the above set of inequality constraints.

\subsection{Kinematic synthesis of leg-wheel subsystem with variable-length crank link}

Dimensional/ Kinematic synthesis is the process of generating the geometry of the mechanism that will perform a specific task. Here, Precision Point Synthesis (PPS) is coupled with optimization to realize a desired path for the wheel-axle of the four-bar leg-wheel subsystem with variable-length crank. Emphasis is placed both on satisfying the kinematic and static specifications in both exactly at precision points in the least squares sense elsewhere.

The calculation of geometric parameters of articulated mechanical or robotic system so that it passes a link in a number of specified successive location or precision points in the plane is referred as Geometric Design Problem [24]. The minimum number of precision points usable for synthesis is limited by the number of loop closure equations 
(also called design equations). Exact synthesis methods guide a point of interest exactly through the specified precision points but solutions exist only if the number of independent design equations is less than (or equal to) the number of design parameters. On the other hand, approximate synthesis methods minimize the structural error to ensure leastsquares satisfaction of the desired specification. This method is mainly used in over-determined geometric design problems where more precision points are defined.

Finding a solution for the four or more precision-point-synthesis problems involve nonlinear equations, hence we consider only the three precision point cases (points $p_{1}, p_{2}$ and $p_{3}$ in Fig. 5). The constraint equations used for 3PPS kinematic synthesis approach are derived from the loop-closure equation for each dyad of the leg-wheel subsystem shown in Fig. 5 as:

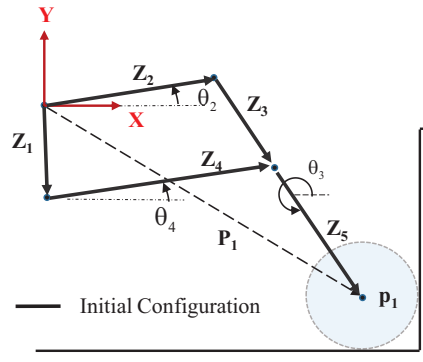

(a)

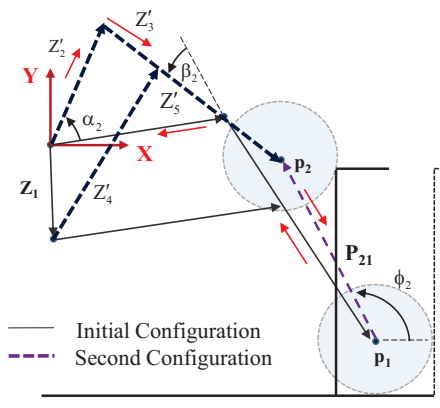

(b)

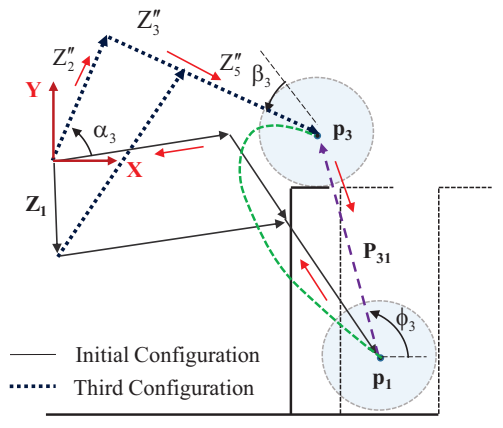

(c)

Figure 5: Kinematic synthesis with three precision points (a) Initial configuration, (b) Second configuration, (c) Third configuration.

$$
\begin{aligned}
\mathbf{Z}_{\mathbf{2}}\left(\rho_{2} e^{i \alpha_{2}}-1\right)+\mathbf{Z}_{\mathbf{6}}\left(e^{i \beta_{2}}-1\right) & =\mathbf{P}_{\mathbf{2}}-\mathbf{P}_{\mathbf{1}} \\
\mathbf{Z}_{\mathbf{2}}\left(\rho_{3} e^{i \alpha_{3}}-1\right)+\mathbf{Z}_{\mathbf{6}}\left(e^{i \beta_{3}}-1\right) & =\mathbf{P}_{\mathbf{3}}-\mathbf{P}_{\mathbf{1}} \\
\mathbf{Z}_{\mathbf{4}}\left(e^{i \gamma_{2}}-1\right)+\mathbf{Z}_{\mathbf{5}}\left(e^{i \beta_{2}}-1\right) & =\mathbf{P}_{\mathbf{2}}-\mathbf{P}_{\mathbf{1}} \\
\mathbf{Z}_{\mathbf{4}}\left(e^{i \gamma_{3}}-1\right)+\mathbf{Z}_{\mathbf{5}}\left(e^{i \beta_{3}}-1\right) & =\mathbf{P}_{\mathbf{3}}-\mathbf{P}_{\mathbf{1}}
\end{aligned}
$$

where $\mathbf{Z}_{\mathbf{6}}=\mathbf{Z}_{\mathbf{3}}+\mathbf{Z}_{\mathbf{5}}$ and $\rho_{2}$ and $\rho_{3}$ are defined as the crank link length stretch ratio of four-bar mechanism when the leg-wheel change from the first to the second precision point, and the second to the third, respectively. Vectors $\mathbf{P}_{\mathbf{1}}, \mathbf{P}_{\mathbf{2}}$ and $\mathbf{P}_{\mathbf{3}}$ are precision points of leg-wheel end-effector which they are determined by problem description (designer has a freedom in selecting the number and location of the precision points), $\mathbf{Z}_{\mathbf{1}}, \mathbf{Z}_{\mathbf{2}}, \mathbf{Z}_{\mathbf{3}}, \mathbf{Z}_{\mathbf{4}}$ and $\mathbf{Z}_{\mathbf{5}}$ are the vectors corresponding to each link of the four-bar and $\mathbf{Z}_{\mathbf{i}}^{\prime}$ and $\mathbf{Z}_{\mathbf{i}}^{\prime \prime}, i=\{1,2,3,4,5\}$ are length of the links in second and third configurations as shown in Fig. 5.

The end point of the four-bar is now constrained to pass through each of the selected precision points on the trajectory. Now, we can rewrite Eq. (11) in the matrix form as follows:

$$
\left[\begin{array}{cccc}
e^{i \gamma_{2}}-1 & e^{i \beta_{2}}-1 & 0 & 0 \\
e^{i \gamma_{3}}-1 & e^{i \beta_{3}}-1 & 0 & 0 \\
0 & 0 & \rho_{2} e^{i \alpha_{2}}-1 & e^{i \beta_{2}-1} \\
0 & 0 & \rho_{3} e^{i \alpha_{3}}-1 & e^{i \beta_{3}}-1
\end{array}\right]\left[\begin{array}{l}
\mathbf{Z}_{\mathbf{4}} \\
\mathbf{Z}_{\mathbf{5}} \\
\mathbf{Z}_{\mathbf{2}} \\
\mathbf{Z}_{\mathbf{6}}
\end{array}\right]=\left[\begin{array}{c}
\mathbf{P}_{2}-\mathbf{P}_{\mathbf{1}} \\
\mathbf{P}_{3}-\mathbf{P}_{\mathbf{1}} \\
\mathbf{P}_{\mathbf{2}}-\mathbf{P}_{\mathbf{1}} \\
\mathbf{P}_{3}-\mathbf{P}_{\mathbf{1}}
\end{array}\right]
$$

Equation (12) contains a total 8 scalar constraints obtained from the two scalar loop-closures per precision point in the path-following problem for each dyad with 16 scalar unknowns which they are $\mathbf{Z}_{\mathbf{1}}=\left(Z_{1 x}, Z_{1 y}\right), \mathbf{Z}_{\mathbf{2}}=$ $\left(Z_{2 x}, Z_{2 y}\right), \mathbf{Z}_{3}=\left(Z_{3 x}, Z_{3 y}\right), \mathbf{Z}_{\mathbf{4}}=\left(Z_{4 x}, Z_{4 y}\right), \alpha_{2}, \alpha_{3}, \gamma_{2}, \gamma_{3}, \beta_{2}, \beta_{3}, \rho_{2}, \rho_{3}$. The stretch ratios $\rho_{2}, \rho_{3}$ are selected as the free choices due to the obvious physical interpretation of the extensibility of an crank link. By selecting $\alpha_{2}, \alpha_{3}, \beta_{2}, \beta_{3}, \gamma_{2}, \gamma_{3}$ as free choices, and $P_{1}, P_{2}$ and $P_{3}$ a linear solution of the equations can be obtained from Eq. (12). 
In general, the desired kinematic motion curve consists of both the motion of the axle with respect to the chassis and the motion of the body with respect to the inertial frame. However, without loss of generality, we will also nominally assume the fixed chassis for the rest of this paper, we will assume step profile move toward the vehicle as illustrated in Fig. 5.

To optimize the discrepancy between the desired and actual curve, each curve should be divided into same length segments/points, $N_{c}$, and calculate their distance. The objective function is taken to be the structural error between the desired $P_{k}$ and actual path $Q_{k}$ computed using arc length based correspondence points. The overall design problem can be expressed in the form of constrained problem as:

$$
\min \sum_{k=1}^{N_{c}}\left(\left(P_{x k}-Q_{x k}\right)^{2}+\left(P_{y k}-Q_{y k}\right)^{2}\right)
$$

subject to

$$
\begin{gathered}
{\left[\begin{array}{l}
\mathbf{Z}_{\mathbf{4}} \\
\mathbf{Z}_{\mathbf{5}} \\
\mathbf{Z}_{\mathbf{2}} \\
\mathbf{Z}_{\mathbf{6}}
\end{array}\right]=\left[\begin{array}{cccc}
e^{i \gamma_{2}}-1 & e^{i \beta_{2}}-1 & 0 & 0 \\
e^{i \gamma_{3}}-1 & e^{i \beta_{3}}-1 & 0 & 0 \\
0 & 0 & \rho_{2} e^{i \alpha_{2}}-1 & e^{i \beta_{2}}-1 \\
0 & 0 & \rho_{3} e^{i \alpha_{3}}-1 & e^{i \beta_{3}}-1
\end{array}\right]^{-1}\left[\begin{array}{l}
\mathbf{P}_{\mathbf{2}}-\mathbf{P}_{\mathbf{1}} \\
\mathbf{P}_{\mathbf{3}}-\mathbf{P}_{\mathbf{1}} \\
\mathbf{P}_{\mathbf{2}}-\mathbf{P}_{\mathbf{1}} \\
\mathbf{P}_{\mathbf{3}}-\mathbf{P}_{\mathbf{1}}
\end{array}\right]} \\
\rho_{\text {min }} \leq\left\{\rho_{2}, \rho_{3}\right\} \leq \rho_{\text {max }}, \alpha_{\text {min }} \leq\left\{\alpha_{2}, \alpha_{3}\right\} \leq \alpha_{\max }, \gamma_{\min } \leq\left\{\gamma_{2}, \gamma_{3}\right\} \leq \gamma_{\max }, \beta_{\min } \leq\left\{\beta_{2}, \beta_{3}\right\} \leq \beta_{\max }
\end{gathered}
$$

\subsection{Static synthesis of leg-wheel subsystem with variable-length crank link}

Selecting the optimal value for stiffness of springs is a critical issue here. Very high spring stiffness restricts the forward movement of the wheel and tremendously increases the wheel slippage at the rear wheels. Very low spring stiffness, on the other hand, will result high spring deformation and significantly improve the no-slip margin. However, there is physical limitation on spring deformation. When traction forces from the rear wheels pushes the front leg toward the obstacle surface, it may deform of the spring and consequently deform the four-bar mechanism until the coupler link (link 3 ) hits the obstacle. Thus, finding the optimum value for the spring stiffness is critical issue.

To reach this aim, we need to find total motor torque at the front wheel. The total required motor actuation torque can be derived using the principle of virtual work for a system to be in equilibrium. The actuation torque depends on both the configuration and spring parameters. To reduce this actuation torque with spring assist, both torsion springs (see Fig. 4(b)) and linear zero-free length springs (see Fig. 3(b)) could be used. Torsion springs are embedded at the passive joints and linear spring is attached to the chassis and rocker link (link 4) to reduce the weight effect of the front leg during climbing and also directly affect the wheel-ground contact forces. In the leg-wheel subsystem design with torsion springs, the potential energy of loaded spring can be obtained as

$$
V_{t s}=\frac{1}{2}\left(K_{2}\left(2 \pi+\theta_{2}-\Omega_{2}\right)^{2}+K_{3}\left(\theta_{3}-\theta_{2}-\Omega_{3}\right)^{2}+K_{4}\left(2 \pi+\theta_{4}-\Omega_{4}\right)^{2}+K_{5}\left(\theta_{3}-\theta_{4}-\Omega_{5}\right)^{2}\right)
$$

where $\Omega_{i}$ is the initial angular position of each torsion spring which is assembled at each passive joint (refer to Fig. 9).

The potential energy of the links due to gravity, and the potential energy of linear spring, which is replaced instead of crank link, respectively, can be written as follows:

$$
\begin{aligned}
V_{g} & =m_{2} g y_{2}+m_{3} g y_{3}+m_{4} g y_{4} \\
V_{S} & =\frac{1}{2} K_{S} z_{2}^{2}(\rho-1)^{2}
\end{aligned}
$$

where $m_{2}, m_{3}$ and $m_{4}$ are link masses, and $y_{2}, y_{3}$ and $y_{4}$ are the height of mass center of the links in XY frame of reference, and $K_{S}$ is the stiffness of the linear spring replaced instead of the crank link. 
Therefore, the total virtual work done on the leg-wheel subsystem with torsion spring assist is written as

$$
\delta W=F_{x} \delta x+F_{y} \delta y+M_{z} \delta \phi-\delta V_{g}-\delta V_{t s}-\delta V_{S}
$$

Substituting the expressions for $\delta x, \delta y, \delta \phi, \delta y_{2}, \delta y_{3}, \delta y_{4}, F_{x}, F_{y}$ and $M_{z}$ from Eqs. (3)-(9) into Eq. (18), the expression for traction force $T$ and normal force $N$ at the front wheel can be found. The final expression for $T$ and $N$ can be rewritten as a linear function of parameter vector $\Gamma$ which is including spring constants and initial angular positions of the torsion springs as

$$
\begin{aligned}
& T=\Delta_{1}\left(\theta_{2}, \rho\right) \Gamma+\Delta_{2}\left(\theta_{2}, \rho\right) \\
& N=\Delta_{3}\left(\theta_{2}, \rho\right) \Gamma+\Delta_{4}\left(\theta_{2}, \rho\right)
\end{aligned}
$$

where $\Delta_{1}, \Delta_{3}, \Delta_{2}, \Delta_{4}$ are functions of generalized coordinates $\theta_{2}$ and $\rho$, and $\Gamma$ is the parameter vector

$$
\Gamma=\left[\begin{array}{lllllllll}
K_{2} & \Omega_{2} K_{2} & K_{3} & \Omega_{3} K_{3} & K_{4} & \Omega_{4} K_{4} & K_{5} & \Omega_{5} K_{5} & K_{S}
\end{array}\right]^{T}
$$

As we mentioned earlier, the goal is maximizing the traction forces of controllable torque input of the vehicle which is equivalent to minimizing the function $\max \left(\frac{T}{N}\right)$ for the front wheel. Therefore, substituting the traction and normal force from Eqs. (19) into Eq. (10) yields

$$
\min \left(\max \left(\frac{\Delta_{1}\left(\theta_{2}, \rho\right) \Gamma+\Delta_{2}\left(\theta_{2}, \rho\right)}{\Delta_{3}\left(\theta_{2}, \rho\right) \Gamma+\Delta_{4}\left(\theta_{2}, \rho\right)}\right)\right)
$$

Here, we only have nine design variables in parameter vector $\Gamma$ which need to be calculated from optimization process of leg-wheel subsystem. Equation (21) includes nine constants, and gives us a freedom for selection of spring constants and preloads. Spring preloads can be selected as free variables and after optimization, the rest of variables will be obtained.

\subsection{Kinetostatic optimization of the leg-wheel subsystem with variable-length crank}

In the kinetostatic optimization, kinematic and static synthesis is combined in optimization problem, and obtain both kinematic and static parameters. A variety of objective functions can be employed for minimization. Here, the weighted sum of the end-effector discrepancy between the desired curve and actual curve, and the virtual friction coefficient minimization, are combined as a final objective function in kinetostatic optimization as

$$
\min \left(\Phi_{S}\left(\max \left(\frac{\Delta_{1}\left(\theta_{2}, \rho\right) \Gamma+\Delta_{2}\left(\theta_{2}, \rho\right)}{\Delta_{3}\left(\theta_{2}, \rho\right) \Gamma+\Delta_{4}\left(\theta_{2}, \rho\right)}\right)\right)+\Phi_{K} \sum_{k=1}^{N_{c}}\left(\left(P_{x k}-Q_{x k}\right)^{2}+\left(P_{y k}-Q_{y k}\right)^{2}\right)\right)
$$

where $\Phi_{S}$ and $\Phi_{K}$ are weighting scalars.

Subject to a set of inequality constraints given by:

(i) $N>0$, which corresponds to the constraints which the wheel always maintains in contact with ground.

(ii) $T \leq \mu_{0} N$ which corresponds to no-slip constraints.

(iii) $\tau_{\text {motor }}^{\text {min }} \leq T R \leq \tau_{\text {motor }}^{\max }$, which corresponds to a torque required to generate the traction must be between $\tau_{\text {motor }}^{\min }$ and $\tau_{\text {motor }}^{\max }$.

As illustrated in Graphical User Interface (GUI) made for leg-wheel subsystem design, the input variables in the kinetostatic optimization include: wheel radius, vehicle total mass, link masses, step profile height, desired kinematic precision points and desired kinematic path. The output of kinetostatic optimization are kinematic parameters including link vectors, torsion/linear spring stiffness, optimum spring attachment points and spring initial configuration. Figure 7(b) shows the crank link length stretch ratio and angle variation over time, and required torque at the front leg wheel with/without torsion spring assist. Figure 7(b) shows that the amount of required torque at the wheel-axle when using torsion springs are significantly less than a design without torsion springs. Table 1 shows the optimal spring constants, initial angular position and link length of leg-wheel subsystem with variable-length crank. 


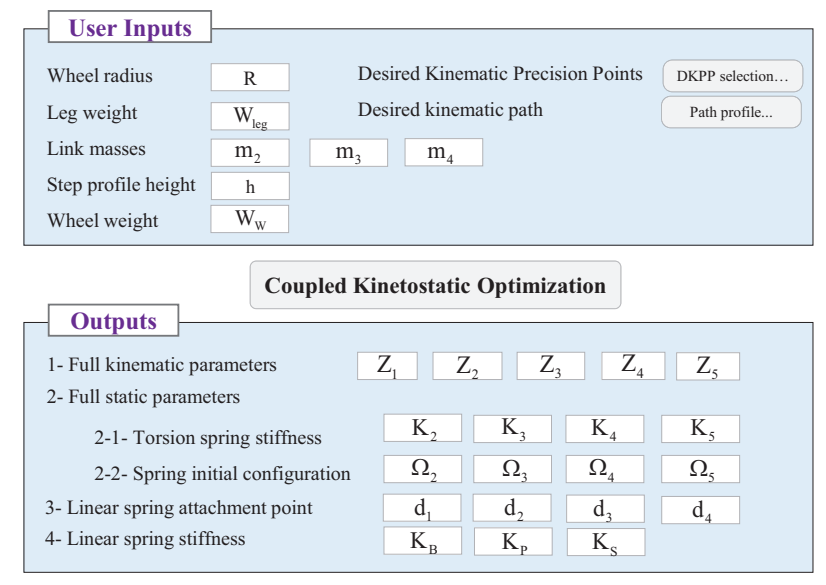

Figure 6: Graphical User Interface (GUI) for kinetostatic optimization.

Table 1: Optimal torsion spring and link length parameters of variable-length crank link

\begin{tabular}{ccc}
\hline \hline Torsion spring constants(N.m/rad) & Initial angular position (rad) & Link length (mm) \\
\hline$K_{2}=60, K_{3}=-51.51, K_{4}=-51.57$ & $\Phi_{2}=0.29, \Phi_{3}=0.36, \Phi_{4}=0.34$ & $z_{1}=46.69, z_{2}=109.60, z_{3}=41.05$ \\
$K_{5}=60, K_{S}=15(N / m)$ & $\Phi_{5}=0.38$ & $z_{4}=134.33, z_{5}=160.32$ \\
\hline
\end{tabular}

The simulation time for passing an obstacle is around eight seconds and the following scenario of the motion is considered for the leg-wheel while passing an obstacle. As illustrated in Fig. 5(a), the initial configuration of leg-wheel is in contact with standard step which its size is twice of wheel's diameter (refer to Fig. 3(a)). We know that the forces and moments at the end-effector are depend upon the motor torques applied on the wheel-axle and chassis' weight. The generated forces from the rear wheels cause a horizontal force which pushes the front wheel toward the obstacle with normal force $N$. When the front wheel is climbing the obstacle, the normal reaction force from the obstacle increases, and consequently no-slip margin will be increased, and then larger values of torques must be commanded to the motors as illustrated in Fig. 7(b). When the front wheel rotates and moves forward, it deforms springs. The deformation and reconfiguration of springs generates additional reaction forces on the surface. These additional normal forces increase the risk of slippage in the rear wheels. In the optimization problem, we aim to minimize the possibility of this slippage. The result of optimization leads to optimum values for geometric and static parameters as shown in Table 1.

\section{Alternate Leg-Wheel Subsystem Designs}

We examine the process by which we can make small change to Eq. (11) to allow for creation of alternate design variants as illustrated in Fig. 8. Figures 8(a) and 8(b) i.e., semi-active crank link designs which are partially balanced with linear and torsional springs, respectively, are primary leg-wheel designs were investigated in section 3 in detail. The amount of motor torque with torsion springs assist in Fig. 7(b) can be compared with the motor torque of other designs illustrated in Figs. 8(c)-8(h). A selected design with the minimum torque requirement can be an appropriate candidate for the leg-wheel subsystem. 


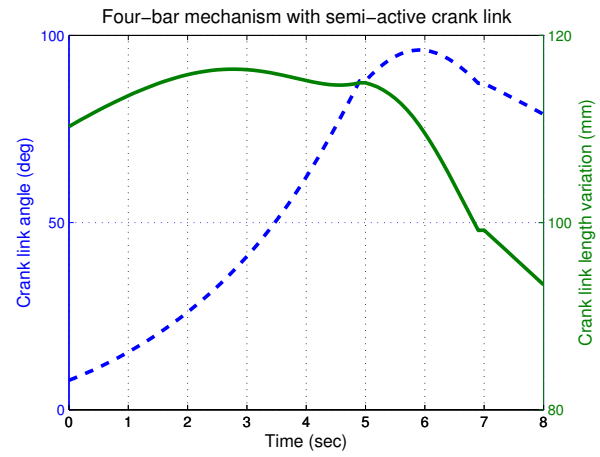

(a)

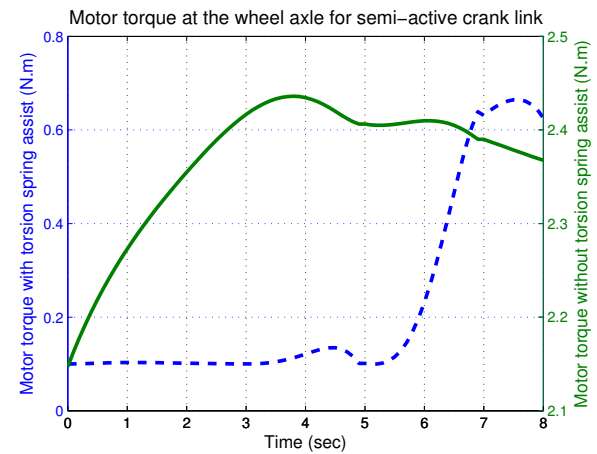

(b)

Figure 7: Kinetostatic synthesis of semi-active leg-wheel subsystem with variable crank link: (a) Crank link-length and angle variation, (b) Torque at the wheel-axle with/without spring assist

\subsection{Semi-active ground link, partially balanced with torsion springs}

As shown in Fig. 8(f), each prescribed precision point vector $\mathbf{P}_{\mathbf{1}}, \mathbf{P}_{\mathbf{2}}$ and $\mathbf{P}_{\mathbf{3}}$ can be expressed as the summation of vectors $\mathbf{Z}_{\mathbf{1}}, \mathbf{Z}_{\mathbf{4}}$ and $\mathbf{Z}_{\mathbf{5}}$ at the corresponding precision points. The kinematic synthesis Eq. (11) can be rewritten as:

$$
\begin{aligned}
\mathbf{Z}_{\mathbf{2}}\left(e^{i \alpha_{2}}-1\right)+\mathbf{Z}_{\mathbf{6}}\left(e^{i \beta_{2}}-1\right) & =\mathbf{P}_{\mathbf{2}}-\mathbf{P}_{\mathbf{1}} \\
\mathbf{Z}_{\mathbf{2}}\left(e^{i \alpha_{3}}-1\right)+\mathbf{Z}_{\mathbf{6}}\left(e^{i \beta_{3}}-1\right) & =\mathbf{P}_{\mathbf{3}}-\mathbf{P}_{\mathbf{1}} \\
\left(\lambda_{2}-1\right) \mathbf{Z}_{\mathbf{1}}+\mathbf{Z}_{\mathbf{4}}\left(e^{i \gamma_{2}}-1\right)+\mathbf{Z}_{\mathbf{5}}\left(e^{i \beta_{2}}-1\right) & =\mathbf{P}_{\mathbf{2}}-\mathbf{P}_{\mathbf{1}} \\
\left(\lambda_{3}-1\right) \mathbf{Z}_{\mathbf{1}}+\mathbf{Z}_{\mathbf{4}}\left(e^{i \gamma_{3}}-1\right)+\mathbf{Z}_{\mathbf{5}}\left(e^{i \beta_{3}}-1\right) & =\mathbf{P}_{\mathbf{3}}-\mathbf{P}_{\mathbf{1}}
\end{aligned}
$$

where $\lambda_{2}$ and $\lambda_{3}$ denote the ground link length stretch ratio when leg-wheel change from first to the second precision point and second to third, respectively. In this design, the total virtual work done on the leg-wheel is the same as Eq. (18).

Figure 9(a) shows the ground link length variation, and Fig. 9(b) shows the required torque at the wheel-axle of leg-wheel subsystems, designed with/without spring assist, over the simulation time. Table 2 shows the optimal spring constants, initial angular positions and link lengths of leg-wheel subsystems with variable-length ground link.

Table 2: Optimal torsion spring and link length parameters of variable-length ground link

\begin{tabular}{ccc}
\hline \hline Torsion spring constants(N.m/rad) & Initial angular position (rad) & Link length (mm) \\
\hline$K_{2}=10.47, K_{3}=0.83, K_{4}=13.7$ & $\Phi_{2}=0.21, \Phi_{3}=4.43, \Phi_{4}=0.07$ & $z_{1}=36.62, z_{2}=91.55, z_{3}=34$ \\
$K_{5}=1.25, K_{S}=48.18(\mathrm{~N} / \mathrm{m})$ & $\Phi_{5}=0.46$ & $z_{4}=115, z_{5}=145.96$ \\
\hline
\end{tabular}

\subsection{Semi-active rocker link, partially balanced with linear spring}

In this design as shown in Fig. 8(c), the constraint equations for 3PPS kinematic synthesis in Eq. (11) only require modification of stretch ratio associated with rocker link length variation:

$$
\begin{aligned}
& \mathbf{Z}_{\mathbf{4}}\left(\eta_{2} e^{i \gamma_{2}}-1\right)+\mathbf{Z}_{\mathbf{5}}\left(e^{i \beta_{2}}-1\right)=\mathbf{P}_{\mathbf{2}}-\mathbf{P}_{\mathbf{1}} \\
& \mathbf{Z}_{\mathbf{4}}\left(\eta_{3} e^{i \gamma_{3}}-1\right)+\mathbf{Z}_{\mathbf{5}}\left(e^{i \beta_{3}}-1\right)=\mathbf{P}_{\mathbf{3}}-\mathbf{P}_{\mathbf{1}}
\end{aligned}
$$




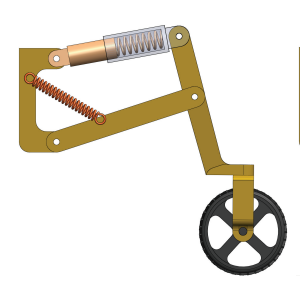

(a)

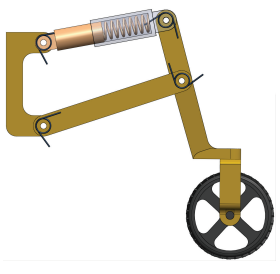

(b)

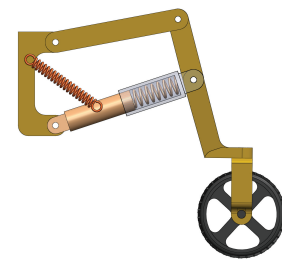

(c)

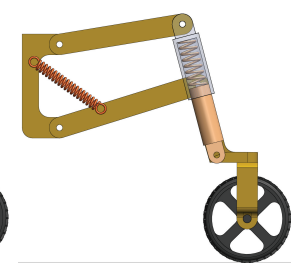

(d)

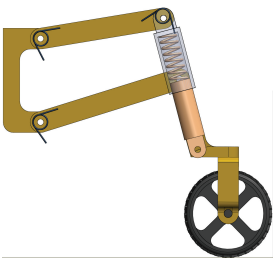

(e)

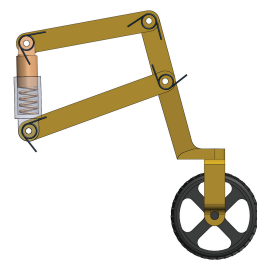

(f)

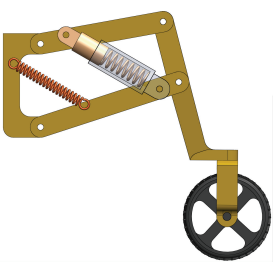

(g)

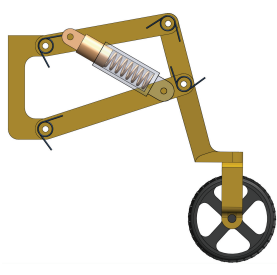

(h)

Figure 8: (a) Semi-active crank link which is partially balanced with linear spring, (b) Semi-active crank link which is partially balanced with torsion springs, (c) Semi-active rocker link which is partially balanced with linear spring, (d) Semi-active coupler link which is partially balanced with linear spring, (e) Semi-active coupler link which is partially balanced with torsion springs, (f) Semi-active ground link which is partially balanced with torsion springs, (g) Fixed-length four-bar with a linear spring in suspension system for wheel-ground contact assurance and linear spring for partially balancing the leg-wheel, (h) Fixed-length four-bar which is partially balanced with torsion springs

where $\eta_{2}$ and $\eta_{3}$ denote the rocker link length stretch ratio when the leg-wheel changes from first to the second precision point and second to third, respectively. In this design, the total virtual work done on the leg-wheel is the same as Eq. (28).

As noticed earlier, another method for partially reducing the front leg-wheel weight effect is using linear springs as shown in Fig. 3(b). In this design, the total potential energy can be written as

$$
V_{l s}=\frac{1}{2} K_{B}\left(d_{3}^{2}+d_{4}^{2}-2 d_{3} d_{4} \sin \left(\theta_{4}\right)\right)
$$

where $K_{B}$ is the stiffness of the linear spring, and $d_{3}$ and $d_{4}$ are the distance from spring attachment points on the link to the rocker link revolute joint.

Figure 10(a) shows the rocker link length and angle variations and, Fig. 10(b) shows the required torque at the wheel-axle of leg-wheel subsystems, designed with/without spring assist, over the simulation time. Table 3 shows the optimal spring constants, initial angular positions and link lengths of leg-wheel subsystems with variable-length rocker link.

\begin{tabular}{|c|c|}
\hline Spring constants $(\mathrm{N} / \mathrm{m})$ & Link length (mm) \\
\hline $\begin{array}{c}K_{S}=20.03 \\
K_{B}=80\end{array}$ & $\begin{array}{c}z_{1}=44.96, z_{2}=110.38, z_{3}=39.10 \\
z_{4}=134, z_{5}=162.13\end{array}$ \\
\hline
\end{tabular}

\subsection{Fixed-length four-bar, partially balanced with linear spring}

In this design (see Fig. 8(g)), the kinematic synthesis Eq. (11) can be rewritten as

$$
\begin{aligned}
& \mathbf{Z}_{\mathbf{2}}\left(e^{i \alpha_{2}}-1\right)+\mathbf{Z}_{\mathbf{6}}\left(e^{i \beta_{2}}-1\right)=\mathbf{P}_{\mathbf{2}}-\mathbf{P}_{\mathbf{1}} \\
& \mathbf{Z}_{\mathbf{2}}\left(e^{i \alpha_{3}}-1\right)+\mathbf{Z}_{\mathbf{6}}\left(e^{i \beta_{3}}-1\right)=\mathbf{P}_{\mathbf{3}}-\mathbf{P}_{\mathbf{1}}
\end{aligned}
$$




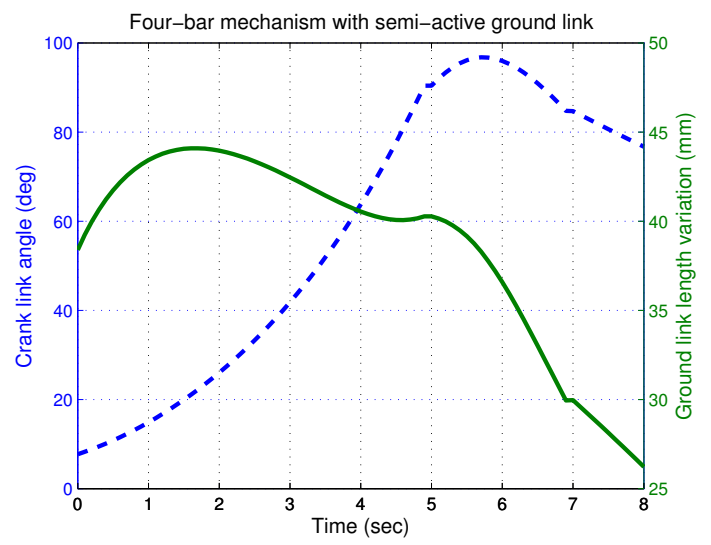

(a)

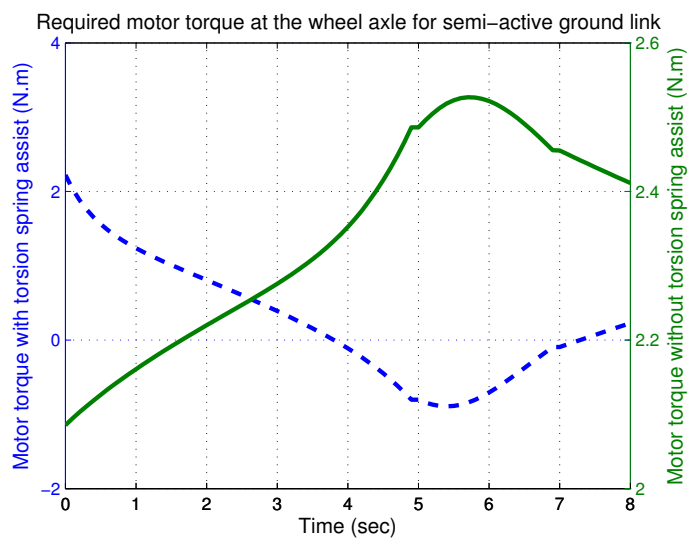

(b)

Figure 9: Kinetostatic synthesis of semi-active leg-wheel subsystem with variable ground link: (a) Ground link-length and crank link angle variation, (b) Torque at the wheel-axle

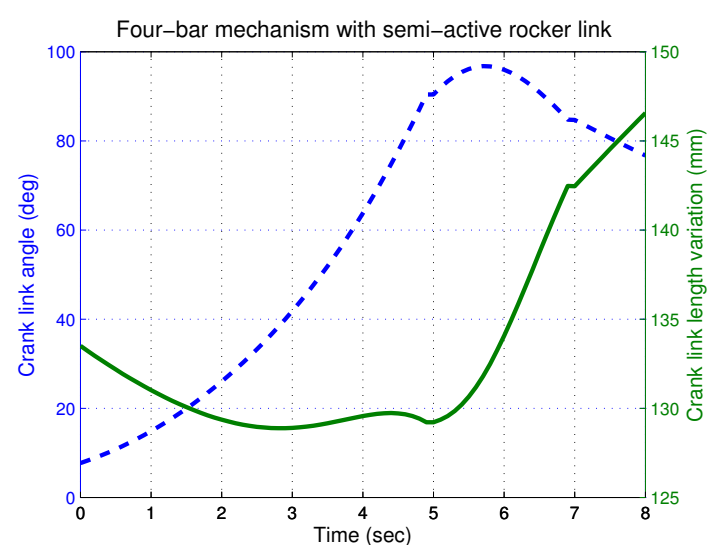

(a)

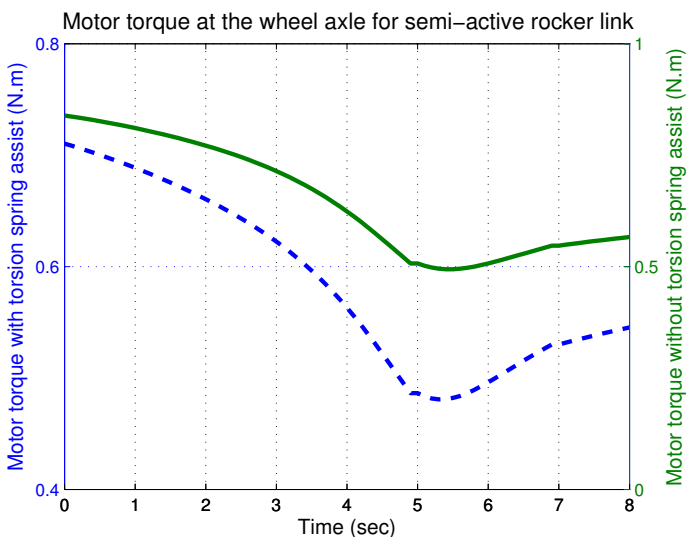

(b)

Figure 10: Kinetostatic synthesis of semi-active leg-wheel subsystem with variable rocker link: (a) Rocker link-length and angle variation, (b) Torque at the wheel-axle

It is noteworthy to mention that in the fixed-length four-bar leg-wheel designs, in order to guarantee the front wheel contact with ground, a linear spring with stiffness $K_{P}$ and free length $l_{0}$ can be placed in suspension system, and can be attached to crank and rocker links from two ends (see Fig. 11(a)). The potential energy stored in this linear spring in the front leg, and also the total virtual work done in this type of leg-wheel design, can be obtained as

$$
\begin{aligned}
& \delta V_{P}=\frac{1}{2} K_{P}\left(l-l_{0}\right)^{2} \\
& \delta W=F_{x} \delta x+F_{y} \delta y+M_{z} \delta \phi-\delta V_{P}-\delta V_{g}-\delta V_{l s}
\end{aligned}
$$

where $\delta l$ is the virtual deflection of the linear spring and can be obtained as

$$
\delta l=\left(d_{1}-d_{2} C_{2}\right) \delta \alpha
$$

where $C_{2}$ is obtained from

$$
\left[\begin{array}{l}
C_{1} \\
C_{2}
\end{array}\right]=\left[\begin{array}{cc}
z_{3} \sin \left(\theta_{3}\right) & -z_{4} \sin \left(\theta_{4}\right) \\
-z_{3} \cos \left(\theta_{3}\right) & z_{4} \cos \left(\theta_{4}\right)
\end{array}\right]^{-1}\left[\begin{array}{c}
-z_{2} \sin \left(\theta_{2}\right) \\
z_{2} \cos \left(\theta_{2}\right)
\end{array}\right] \delta \alpha
$$


Table 4: Optimal torsion spring and link length parameters of fixed-length four-bar leg-wheel

\begin{tabular}{ccc}
\hline \hline Torsion spring constants(N.m/rad) & Initial angular position (rad) & Link length (mm) \\
\hline$K_{2}=-24.91, K_{3}=11.68, K_{4}=-4.45$ & $\Phi_{2}=-0.36, \Phi_{3}=-4.70, \Phi_{4}=-0.15$ & $z_{1}=36.62, z_{2}=91.55, z_{3}=34$ \\
$K_{5}=33.31, K_{S}=15(N / m)$ & $\Phi_{5}=-1.2$ & $z_{4}=115, z_{5}=145.96$ \\
\hline
\end{tabular}

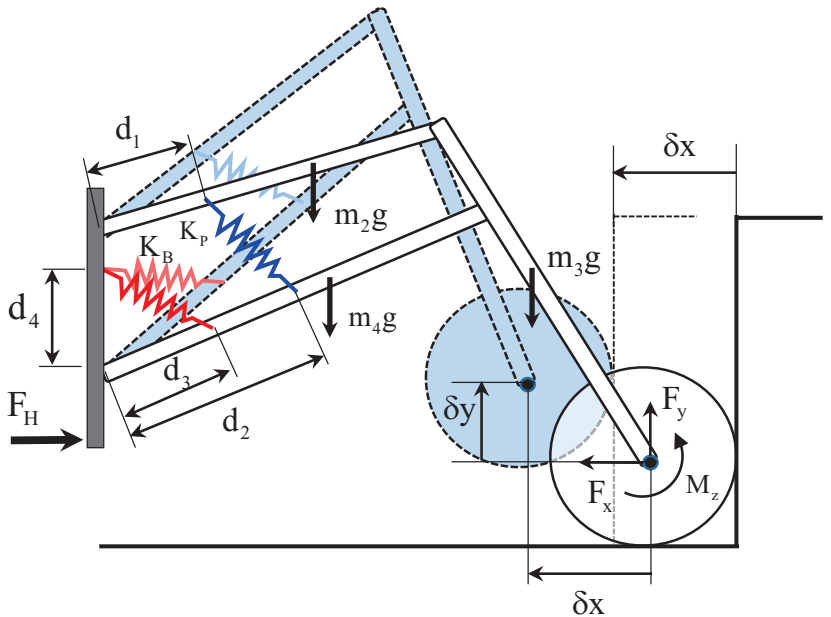

(a)

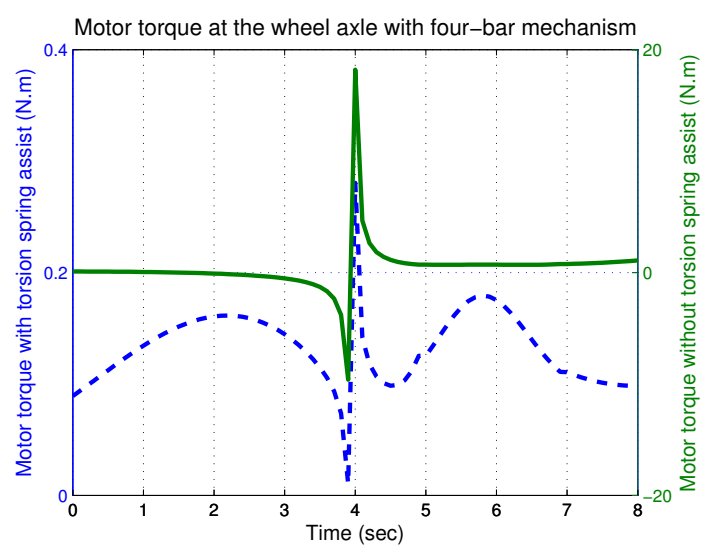

(b)

Figure 11: (a) Four-bar leg-wheel subsystem which is partially balanced with linear spring, and also assure wheel-ground contact with a linear spring in suspension system, (b) Torque at the wheel-axle of leg-wheel subsystem with fixed-length four-bar.

Fig.11(b) shows the required torque at the wheel-axle of leg-wheel subsystems, designed with/without spring assist, over the simulation time. It is clear that the torque with spring assist is significantly less than a design without spring assist. However, because of uneven and spiky changes in motor torque requirement during obstacle surmounting, this design is not preferred. Table 4 shows the optimal spring constants, initial angular positions and link lengths of leg-wheel subsystems with fixed-length four-bar mechanism.

\section{Discussion}

In this paper, we proposed alternate articulated mechanisms for leg-wheel subsystems and presented a general framework and methodology for their kinetostatic design optimization for planar rough terrain traversal tasks. The developed methodology allows the designer to specify a wheel-axle path and prescribe forces along it to accurately match the kinematic and kinetostatic specifications at the precision points and in a least squares sense over the entire range. The kinetostatic design framework is a combination of kinematic design, structural equilibration design and design with spring assists, which permits the designer to arrive at optimal values for the mechanism parameters, as well as the spring parameters simultaneously.

Alternate designs of articulated leg-wheel subsystems were considered to modulate the motion and force interactions between the ground and the chassis, and thereby enable vehicle systems to locomote in difficult environments and rough-terrain. The addition of springs substantially reduces the input torque requirements as demonstrated by extensive simulation studies. The torques along with the stretch ratio for semi-active crank, ground and rocker designs and fixed length designs are shown in Figs. 7-11. In the semi-active ground-link design the actuators can be basemounted but the trade-off is significant torque increase in comparison with semi-active crank and rocker link. Thus, from this perspective, semi-active crank or rocker are better ways to physically realize the actuation torque reduction. 


\section{ACKNOWLEDGMENT}

This work is partially supported by NSF Grant IIS-1319084 and CNS-1314484.

\section{References}

[1] G. Ishigami, K. Iagnemma, J. Overholt, and G. Hudas. "Design, Development, and Mobility Evaluation of an Omnidirectional Mobile Robot for Rough Terrain.", Journal of Field Robotics, 2014, pp. 1-17, doi: 10.1002/rob.21557.

[2] M. Tarokh, G. J. McDermott, "Kinematics modeling and analysis of articulated rovers.", IEEE Transaction on Robotics, Vol. 21 (4), 2005, pp. 539-553.

[3] K. Nagatani, A. Yamasaki, K. Yoshida, T. Adachi, "Development and control method of six-wheel robot with rocker structure.", In Proceedings of the 2007 IEEE International Workshop on Safety, Security and Rescue Robotics, Rome, Italy, 2007.

[4] A. Bouloubasis, G. McKee, P.Tolson, "Novel concepts for a planetary surface exploration rover.", Industrial Robots: An International Journal, Vol. 34 (2), 2007, pp. 116-121.

[5] P. Morin, C. Samson, "Motion control of wheeled mobile robots.", Springer Handbook of Robotics, 2008, pp. 799-826.

[6] S. Kitano, S. Hirose, G. Endo, and E.F. Fukushima. "Development of lightweight sprawling-type quadruped robot TITAN-XIII and its dynamic walking.", In Proceedings of Intelligent Robots and Systems (IROS), IEEE/RSJ International Conference, 2013 , pp. 6025-6030.

[7] S. Nakajima, "RT-Mover: a rough terrain mobile robot with a simple leg-wheel hybrid mechanism.", The International Journal of Robotics Research, Vol. 30 (13), 2011, pp. 1609-1626.

[8] H.W. Stone, "Mars pathfinder microrover: A low-cost, low-power spacecraft.", In Proceedings of the 1996 AIAA Forum on Advanced Developments in Space Robotics, Madison, WI, 1996.

[9] R. Volpe, J. Balaram, T. Ohm, R. Ivlev, "Rocky 7: A next generation Mars rover prototype.", Journal of Advanced Robotics. Vol. 11 (4), 1997, pp. 341-358.

[10] R. Siegwart, P. Lamon, T. Estier, M. Lauria, and R. Piguet, "Innovative Design for Wheeled Locomotion in Rough Terrain.", Robotics and Autonomous Systems, Vol. 40 (2), 2002, pp. 151-162.

[11] G. Besseron, C. Grand, F. Ben Amar, and P. Bidaud. "Decoupled control of the high mobility robot hylos based on a dynamic stability margin." In Proceedings of Intelligent Robots and Systems, IEEE/RSJ International Conference, 2008, pp. 2435-2440.

[12] T. Kubota, Y. Kuroda, Y. Kunii, I. Natakani, "Micro-planetary rover Micro5.", In Proceedings of the Fifth International Symposium on Artificial Intelligence, Robotics and Automation in Space (ESA SP-440), Noordwijk, Netherlands, 1999, pp. $373-378$.

[13] D. Wettergreen, M. Bualat, D. Christian, K. Schwehr, et. al, "Operating Nomad During the Atacama Desert Trek.", In Proceedings of Field and Service Robotics Conference, December 7-10, 1997, Canberra, Australia.

[14] S.V. Sreenivasan, and K.J. Waldron, "Displacement Analysis of an Actively Articulated Wheeled Vehicle Configuration With Extensions to Motion Planning on Uneven Terrain.", ASME Journal of Mechanical Design, Vol. 118 (2), 1996, pp. 312-320.

[15] A. Alamdari, and V. Krovi. "Active Reconfiguration for Performance Enhancement in Articulated Wheeled Vehicles.", In Proceedings of ASME 2014 Dynamic Systems and Control Conference. American Society of Mechanical Engineers, San Antonio, Texas, USA, October 22-24, 2014.

[16] A. Alamdari, X. Zhou, and V. Krovi, "Kinematic Modeling, Analysis and Control of Highly Reconfigurable Articulated Wheeled Vehicles", In Proceedings of the ASME 2013 International Design Engineering Technical Conferences and Computers in Engineering Conference, ASME IDETC/CIE 2013, August 4-7, 2013, Portland, OR.

[17] A. Halme, I. Leppanen, and S. Salmi, "Development of Workpartner- Robot Design of Actuating and Motion Control System.", In Proceedings of 2nd International Conference on Climbing and Walking Robots (CLAWAR 99), Portsmouth, England, Wiley Publishers, September 13-15, 1999, pp. 657-666.

[18] G. Endo, and S. Hirose, "Study on Roller-Walker Multi-Mode Steering Control and Self-Contained Locomotion.", In Proceedings of 2000 IEEE International Conference on Robotics and Automation, San Francisco, CA, April 24-28, 2000, pp. 2808-2814.

[19] M. Hiller, and D. German, "Manoeuvrability of the Legged and Wheeled Vehicle ALDURO in Uneven Terrain with Consideration of Nonholonomic Constraints.", In Proceeding of 2002 International Symposium on Mechatronics (ISOM 2002), March 21-22, 2002, Chemnitz, Germany.

[20] A. Alamdari, and V. Krovi, "Static balancing of highly reconfigurable articulated wheeled vehicles for power consumption reduction of actuators", International Journal of Mechanisms and Robotic Systems, 2016, (In press).

[21] H. Hong, D. Kim, H. Soo Kim, S. Lee, and J. Kim., "Contact angle estimation and composite locomotive strategy of a stair-climbing mobile platform." Robotics and Computer-Integrated Manufacturing, Vol. 29 (5), 2013, pp. 367-381.

[22] A.K. Singh, N. Rahul Kumar, V. Eathakota, and K. K. Madhava. "A novel compliant rover for rough terrain mobility.", In Proceedings of Intelligent Robots and Systems (IROS), IEEE/RSJ International Conference, 2010, pp. 4788-4793.

[23] S. K. Jun, G.D. White, V. Krovi, "Kinetostatic Design Considerations for an Articulated Leg-Wheel Locomotion Subsystem.", Journal of Dynamic Systems, Measurement, and Control, 2006, Vol. 128, pp. 112-121.

[24] Robert L. Norton, "Design of Machinery: An Introduction to the synthesis and analysis of Mechanism and Machines.", McGraw-Hill Inc., USA, 1992.

[25] P. Lamon, A. Krebs,M. Lauria, R. Siegwart, and S. Shooter, "Wheel torque control for a rough terrain rover.", In Proceedings of IEEE International Conference on Robotics and Automation, 2004, Vol. 5, pp. 4682-4687, doi: 10.1109/ROBOT.2004.1302456

[26] A. Alamdari, J. Sovizi, S.K. Jun, V., Krovi, "Kinetostatic Optimization for an Adjustable Four-Bar Based Articulated Leg-Wheel Subsystem", IEEE/RSJ, Intelligent Robots and Systems, 2014, pp. 2860 - 2866, DOI: 10.1109/IROS.2014.6942955. 